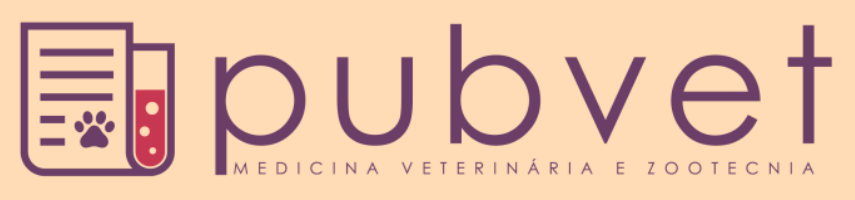

https://doi.org/10.22256/pubvet.v12n3a54.1-6

\title{
Diagnóstico e tratamento de lúpus eritematoso discoide canino: Relato de caso
}

\author{
Vanessa Lucas da Silva ${ }^{01 *}$, Cynthia Levi Baratta Monteiro ${ }^{2}$, Michelle Costa e Silva ${ }^{\bullet}$, \\ Raimundo Diones Carneiro ${ }^{\bullet}$, Euclides R Pereira Junior ${ }^{\oplus}$, Lorena Vasconcelos de Lucena ${ }^{\circ} 5$ \\ ${ }^{I}$ Discente do Curso de Medicina Veterinária - Faculdade Terra Nordeste (FATENE) - E-mail: vanessaluc@ gmail.com *Autor para correspondência \\ ${ }^{2}$ Professora da Faculdade Terra Nordeste (FATENE), Departamento de Medicina Veterinária. Caucaia - CE Brasil - cyntia.baratta@fatene.edu.br \\ ${ }^{3}$ Professora da Faculdade Terra Nordeste (FATENE), Departamento de Medicina Veterinária. Caucaia - CE Brasil-E-mail:michelle.silva@fatene.edu.br \\ ${ }^{4}$ Médico Veterinário da Policlínica Veterinária Metropolitana de Caucaia (Fortaleza, Ceará, Brasil) - E-mail:diones.carneiro@fatene.edu.br \\ ${ }^{5}$ Médico Veterinário da Policlínica Veterinária Metropolitana de Caucaia (Fortaleza, Ceará, Brasil) -E-mail: -E-mail:euclidesrpjmedvet@hotmail.com \\ ${ }^{6}$ Discente do Curso de Medicina Veterinária - Faculdade Terra Nordeste (FATENE) - E-mail: lorennavasconcelos@yahoo.com.br
}

RESUMO. O complexo Lúpus Eritematoso (LE) é constituído por um conjunto de dermatopatias autoimunes que pode se manifestar em duas apresentações clinicas principais: o Lúpus Eritematoso Sistêmico (LES) e o Lúpus Eritematoso Discóide (LED). O diagnóstico clínico do LED muitas vezes é um desafio, devido à variedade de sintomas que pode ocorrer tanto no lúpus, como em outras patologias dermatológicas, fato este que interfere na rapidez do diagnóstico e consequentemente no tratamento e melhora do paciente. Este trabalho tem como objetivo relatar a importância do exame histopatológico no diagnóstico definitivo para o tratamento e prognóstico favorável de paciente canino, poodle com LED. Foi atendido na Policlínica veterinária metropolitana - HVM, um cão da raça poodle, com lesão em região do focinho tendo passado por tratamentos anteriores sem resultado aparente, foram feitos exames laboratoriais e citológicos para que fossem descartadas outras dermatopatias. Após resultado inconclusivo na citologia, foi solicitado o histopatológico, para definição do diagnóstico exato e exclusão de outras dermatopatias. O histopatológico confirmou o diagnóstico de Lúpus Eritematoso Discóide. Foi instituído tratamento com corticosteroides na dose de $1,0 \mathrm{mg} / \mathrm{kg}$, vitaminas antioxidantes e protetor solar. Após 10 dias houve o aumento da dose para $2,5 \mathrm{mg} / \mathrm{kg}$, que é a dose imunossupressora. $\mathrm{O}$ animal apresentou resposta com diminuição da região crostosa da lesão em plano nasal.

Palavras chave: dermatopatias, doença crônica, poodle.

\section{Diagnosis and treatment of canine discoid lupus erythematosus: Case report}

ABSTRACT. The Lupus Erythematosus immune complex disease (LE) consists of a set of autoimmune dermatopathies that can manifest in two main clinical presentations: Systemic Lupus Erythematosus (SLE) and Discoid Lupus Erythematosus (DLE). The clinical diagnosis of DLE can be challenging, due to the variety of symptoms that can occur both in lupus and in other dermatological conditions, which interferes with the early diagnosis and consequently a delay in the treatment and improvement of the patient. This study aims to report the importance of histopathological examination in the definitive diagnosis for treatment and favorable prognosis of canine poodle with DLE. A poodle dog was attended at the Metropolitan Veterinary Polyclinic - HVM, presenting lesion in nasal area with previous treatments and no apparent improvment of the lesion. Laboratory and cytological exams were done to exclude other skin diseases. After an inconclusive result in the cytology, the histopathological examination was requested, to 
define the exact diagnosis and exclusion of other dermatopathies. The histopathological confirmed the diagnosis of Discoid Lupus Erythematosus. Treatment with corticosteroids at the dose of $1.0 \mathrm{mg} / \mathrm{kg}$, antioxidant vitamins and sunscreen was instituted. After 10 days, the dose increased to $2.5 \mathrm{mg} / \mathrm{kg}$, which is the immunosuppressive dose. The animal presented a response with a decrease in the crustal region of the lesion in the nasal plane.

Keywords: dermatopathies, chronic disease, poodle

\section{Diagnóstico y tratamiento de lupus eritematoso discoide canino: Relato de caso}

RESUMEN. El complejo Lupus Eritematoso (LE) está constituido por un conjunto de dermatopatías autoinmunes que puede manifestarse en dos presentaciones clínicas principales: el Lupus Eritematoso Sistémico (LES) y el Lupus Eritematoso Discóide (LED). El diagnóstico clínico del LED a menudo es un desafío, debido a la variedad de síntomas que puede ocurrir tanto en el lupus, como en otras patologías dermatológicas, hecho que interfiere en la rapidez del diagnóstico y consecuentemente en el tratamiento y mejora del paciente. Este trabajo tiene como objetivo relatar la importancia del examen histopatológico en el diagnóstico definitivo para el tratamiento y pronóstico favorable de paciente canino, Poodle con LED. En la Policlínica veterinaria metropolitana - HVM, un perro de la raza Poodle, con lesión en región del hocico, habiendo pasado por tratamientos anteriores sin resultado aparente, se realizaron exámenes de laboratorio y citológico para que fueran descartadas otras dermatopatías. Después de un resultado inconcluso en la citología, se solicitó el histopatológico, para definir el diagnóstico exacto y la exclusión de otras dermatopatías. El histopatológico confirmó el diagnóstico de Lupus Eritematoso Discóide. Se estableció tratamiento con corticosteroides a la dosis de $1,0 \mathrm{mg} / \mathrm{kg}$, vitaminas antioxidantes y protector solar. Después de 10 días hubo el aumento de la dosis a $2,5 \mathrm{mg} / \mathrm{kg}$, que es la dosis inmunosupresora. El animal presentó respuesta con disminución de la región costrosa de la lesión en plano nasal.

Palabras clave: dermatopatías, enfermedad crónica, Poodle

\section{Introdução}

A dermatologia é uma especialidade na medicina veterinária que vem se tornando crescente na clínica de pequenos animais, muitas vezes se tornando um desafio ao clínico, por dificuldades no diagnóstico ou por tratamento longos e que exigem muito do proprietário. Dentre as patologias dermatológicas, as dermatopatias autoimunes, embora raras, tem se tornado de grande importância devido ao difícil diagnóstico e semelhança nos sinais clínicos (Cardoso et al., 2011).

As dermatopatias autoimunes são patologias causadas por uma orientação desordenada do sistema imune dos pacientes acometidos, que faz com que este reaja de forma incomum e anormal contra componentes do seu próprio organismo (Larsson, 1996). Estas têm causa multifatorial, onde se acredita que fatores genéticos e ambientais sejam os mais relevantes.

Dentre estas dermatopatias, o complexo Lúpus Eritematoso (LE) é uma das que tem o diagnóstico mais desafiador devido a especificidade do exame e as características lesionais da doença que se assemelham a grande variedade de doenças dermatológicas. Este é considerado como a segunda doença dermatológica autoimune mais frequente diagnosticada em cães. Embora seja a segunda doença mais frequente, acredita-se que, o número de casos diagnosticados é ainda menor que o real, uma vez que esta doença apresenta um difícil diagnóstico (Scott et al., 1996, Gerhauser et al., 2006). Esta dificuldade em se elucidar um diagnóstico específico pode estar ligada a falta de informação do clínico, relutância do proprietário em tratar lesões similares em doenças mais frequentes e de maior importância zoonótica, subjulgamento de uma doença rara e de difícil achado clínico ou somente, uma junção de todas essas opções. Devido a esses problemas o clínico deve sempre estar disposto a fazer um diagnóstico diferencial e alertar o proprietário sobre possíveis complicações de animais com dermatopatias. 
O complexo Lúpus Eritematoso (LE) é constituído por um conjunto de dermatopatias autoimunes que se manifestam em duas principais apresentações clinicas: o Lúpus Eritematoso Sistêmico (LES) e o Lúpus Eritematoso Discóide (LED), que é a forma benigna do LES, limitada a pele (Tizard, 2002). O LES é uma doença inflamatória crônica de etiologia multifatorial, caracterizada pelo envolvimento de diferentes órgãos e sistemas com desordens imunológicas com autoanticorpos (Gomes, 2015). O LED, também referido como lúpus eritematoso cutâneo crônico (LECC), caracteriza-se por ser uma afecção dermatológica benigna, sendo diferenciado de sua variante sistêmica a partir da ausência de manifestações extras tegumentares (Griffies et al., 2004, Goo et al., 2008). Dentre elas a mais comum em animais é o LED. Sua patogênese ainda não foi bem elucidada, mas sabe-se que, pode haver predisposição genética. Patel \& Forsythe (2011) afirmam que a exposição UV é uma das principais preocupações na doença, em animais predispostos geneticamente, ocorrendo deposição de antígenos/anticorpos na junção dermoepidémica no LED (Lúpus eritematoso discoide) o que induz clinicamente as lesões cutâneas (Gomes, 2015). Larsson \& Otsuka (2000) não verificaram diferença em animais de raça definida $(52,3 \%)$ dos de raça não definida $(47,7 \%)$. No entanto, fora do país, Miller et al. (2013) demonstraram que as principais raças acometidas são Collies, Pastores de Shetland, Pointers Alemães e Huskies siberianos.

No LED observa-se principalmente despigmentação, eritema e descamação do focinho e região distal dos membros, genitais e cavidade oral (Gross et al., 2009). O eritema e a despigmentação observados no plano nasal podem progredir para a formação de crostas e ulcerações. Além disso, pode haver progressão da doença para a ponte nasal, cavidade oral e região periorbitária e nos casos crônicos alopecia e formação de cicatriz (Patel \& Forsythe, 2011).

Em casos de LED o exame histopatológico é imprescindível para o diagnóstico definitivo e prognóstico do paciente. As características histopatológicas em biópsias de pele revelam dermatite de interface, seja hidrópica, liquenóide ou mista, ocorrendo edema intracelular (degeneração hidrópica) da camada basal e inúmeros queratinócitos necrosados na epiderme, já na liquenóide observa-se uma infiltração mononuclear com predomínio plasmocitário adjacente aos vasos e constituintes dermais ( $\underline{\mathrm{Scott}}$ et al., 1996, Patel \& Forsythe, 2011, Val, 2006). Esse predomínio plasmocitário é o que diferencia o lúpus canino ao lúpus humano, já que no humano a predominância é de linfócitos $\mathrm{T}$. O tratamento é baseado no uso de imunossupressores e antibiticoterpia associada quando necessário, além do uso de vitaminas para a eliminação de radicais livres.

Dentre as dermatopatias autoimunes, deve-se fazer diagnóstico diferencial do lúpus com Dermatomiosite Canina Familiar, Complexo Pênfigo, Síndrome Uveodermatológica, dermatite nasal solar e Lúpus eritematoso sistêmico. Mas o LED precisa ser diferenciado de patologias fúngicas (Esporotricose, Criptococose, Malassezia), bacterianas (piodermites), alergias (alimentares ou atopia) e protozoárias (Leishmaniose).

Portanto, o grande desafio desta dermatopatia está na dificuldade de diagnóstico, pela sua sintomatologia generalista, podendo se assemelhar a diversas patologias dermatológicas, o que costuma confundir o clínico, dificultando o tratamento e regressão das lesões. Fazendo com que, o proprietário desacredite no tratamento e busque outras soluções. Por isso, é de grande importância se fazer a análise laboratorial das lesões, seja por citologia para descarte de outras patologias ou por histopatológico para confirmação do diagnóstico. Diante disso, este trabalho tem como objetivo relatar a importância do exame histopatológico no diagnóstico definitivo para o tratamento e prognóstico favorável de paciente canino, poodle, com LED, demonstrando a diversidade da doença não só em a raças predispostas.

\section{Material e Métodos}

No dia dois de agosto de 2017 foi atendido na Policlínica Veterinária Metropolitana - HVM situada em Caucaia- CE, um cão da raça poodle, macho, de 3 anos de idade, com histórico de lesão no plano nasal, de aspecto crostoso e avermelhado (Figura 1). A aproximadamente um mês, o referido animal já tinha sido consultado por outro veterinário, o qual prescreveu somente tratamento tópico, a base de gentamicina, sulfadiazina de prata e vitamina A, por um período de 15 dias, não tendo obtido melhora significativa.

$\mathrm{Na}$ anamnese, o proprietário exibiu o cartão de vacinação do animal com todas principais 
vacinas, vermifugação em dia, controle de ectoparasitas periódico, sem acesso à rua, fezes e urina normais. No exame físico, o animal apresentou temperatura corpórea de $39^{\circ} \mathrm{C}$, com linfonodos submandibulares alterados e demais normais, mucosa oral e oftálmica congestas, tendo o tempo de preenchimento capilar normal, frequência respiratória e cardíaca dentro da normalidade. No momento da consulta foram coletadas amostras de sangue para realização do hemograma completo e dosagens bioquímicas de creatinina e alanina aminotransferase (ALT), além de ser dosada a glicose através de glicosímetro. Também foram coletadas amostras das lesões do plano nasal para exame citológico, com escarificação das mesmas e realização de imprint, onde o material foi disposto em cinco lâminas e enviado ao laboratório de patologia clínica da Policlínica Veterinária Metropolitana.

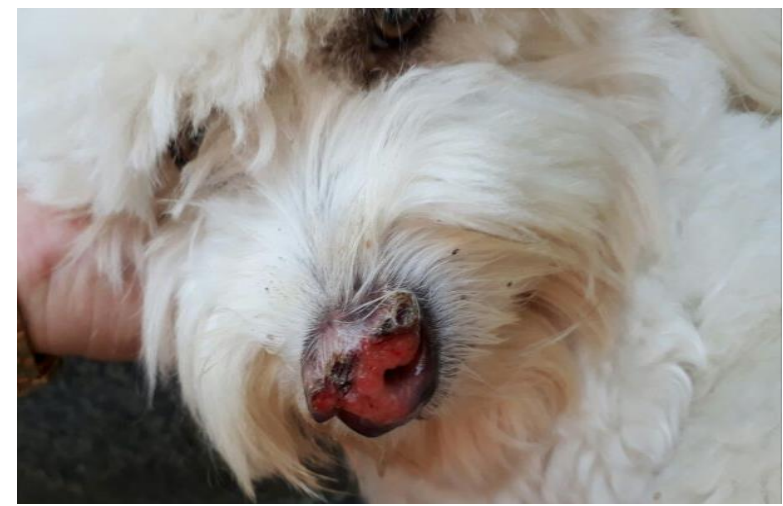

Figura 1. Animal na primeira consulta dia $02 / 08 / 2017$, onde se verifica lesão em plano nasal, com crosta e vermelhidão.

\section{Resultados e Discussão}

Durante atendimento, as lesões restringiam-se ao plano nasal. Está sendo bem caracterizada por ocorrer na transição do plano nasal com a pele hirsuta circunvizinha podendo acometer, concomitantemente, a dobra alar medial e ventral. Assim, promovendo despigmentação da área, assumindo tonalidade acinzentada ou azulada, além da comuníssima perda da arquitetura do plano nasal, adquirindo uma superfície de aparência lisa (Mueller, 2005). O que são aspectos bem característicos, segundo a literatura, de uma dermatopatia autoimune.

Devido à tentativa falha no tratamento tópico anterior, podem-se descartar algumas doenças que teriam êxito com esse tratamento, como as doenças fúngicas, bacterianas ou virais.

De acordo com o laudo do hemograma podese verificar um quadro de linfopenia (Tabela 1). Este fato surpreendeu o clínico, visto que em doenças autoimunes o sistema imunológico tem uma ação exacerbada, o que promove um quadro de linfocitose. No entanto, a exposição à radiação ultravioleta pode contribuir com a alteração da regulação imunológica mediada pelas células $T$, que promove a exacerbação da atividade de linfócitos B e inibição da atividade de linfócitos T supressores (Tizard, 2002) o que explicaria a linfopenia. Esse dado pode coincidir também com alterações de estresse, manejo alimentar ou alguma outra patologia em fase inicial.

Tabela 1. Hemogramas realizados em $02 / 08 / 2017 \quad\left(1^{\circ}\right.$ atendimento) e 29/09/2017 ( $2^{\circ}$ atendimento).

\begin{tabular}{lccc}
\hline Item & $\begin{array}{c}1^{\circ} \\
\text { Atendimento }\end{array}$ & $\begin{array}{c}2^{\circ} \\
\text { Atendimento }\end{array}$ & Referências - Cães C \\
\hline Eritrócitos & 7.800 .000 & 7.300 .000 & $5.500 .000-8.500 .000 / \mathrm{mm}^{3}$ \\
Hemoglobina & 19.0 & 16.8 & $12-18(\mathrm{~g} / \mathrm{dL})$ \\
Hematócrito & 57 & 50 & $37-55(\%)$ \\
VGM & 79.0 & 68.4 & $60-77(\mathrm{fL})$ \\
CHGM & 33.3 & 33.6 & $32-36(\%)$ \\
Plaquetas & 280.000 & 210.000 & $200.000-500.000 / \mathrm{mm} 3$ \\
Proteína Total & 8.2 & 8.0 & $6.0-8.0(\mathrm{~g} / \mathrm{dL})$ \\
Leucócitos & 9.100 & 11.700 & $6.000-17.000 / \mathrm{mm} 3$ \\
Segmentados & 7.826 & 9.594 & $300-11.500 / \mathrm{mm} 3$ \\
Linfócitos & 364 & 936 & $1.000-4.800 / \mathrm{mm} 3$ \\
Eosinófilos & 819 & 1.287 & $100-1.250 / \mathrm{mm} 3$ \\
Monócitos & - & - & $150-1.350 / \mathrm{mm} 3$ \\
Aminotransferase & 40 & 152 & \\
Creatinina & 0,9 & 1,0 & $0,5-1,5 \mathrm{mg} / \mathrm{dL}$
\end{tabular}

$\mathrm{Na}$ análise citológica da lesão do plano nasal foi evidenciada moderada celularidade, constituída predominantemente por neutrófilos, além de células com baixa razão núcleo/citoplasma, citoplasma basofílico. Em coloração pelo Gram foram observados ocasionais cocos Gram-positivos e bastonetes Gram-negativo. Tais observações não sugeriram um diagnóstico conclusivo com relação à causa, mas foi de grande valia para descarte de outras patologias, tendo sido sugerida realização de exame histopatológico.

Para o histopatológico o animal foi direcionado ao laboratório Pathovet - Anatomia Patológica e Patologia Clínica Veterinária, onde foi retirado um fragmento da lesão do plano nasal para posterior análise. No histopatológico foram descritas extensa hiperqueratose e áreas crostosas que recobrem ulceração por processo inflamatório, padrão liquenóide na junção dermoepidérmica se difundindo por toda a derme, constituída por linfócitos, plasmócitos e alguns macrófagos e neutrófilos. Observaram-se áreas 
de necrose e hemorragia, com ausência de sinais de malignidade. De acordo com as alterações observadas foi possível identificar quadro histopatológico compatível com lúpus eritematoso discoide.

Conforme descrito na literatura, o histopatológico é de fundamental importância para definição do correto diagnóstico e instituição do tratamento. A ocorrência na raça em questão diverge da literatura que não cita poodle como frequente ou não foram encontrados na literatura relatos nessa raça. Embora esta raça, não esteja entre as raças que tem maior frequência no diagnóstico, esta tem tido crescente demanda na clínica de pequenos, acredita-se que isso ocorra devido à grande quantidade de animais com esta raça.

Após o resultado do histopatológico o animal foi submetido a tratamento com corticosteroides, em dose menor ao recomendado em doenças imunossupressoras, devido ao baixo valor de células do sistema imunológico. Assim, o tratamento foi iniciado com dose de $1 \mathrm{mg} / \mathrm{kg} / \mathrm{dia}$ de prednisolona, vitamina $\mathrm{E}$ na dose de 400UI/BID, fator de proteção solar 45 em creme com hidrocortisona para uso tópico, BID (Ettinger \& Feldman, 2004). O tratamento foi mantido por um período de 10 dias para análise da resposta imunológica do animal. Pediu-se para que a proprietária voltasse à policlínica após o término do período do tratamento estabelecido para nova avaliação hematológica.

No retorno, observou-se melhora significativa das lesões em plano nasal do animal, de subjetivamente $40 \%$, e satisfação do proprietário. Foi realizado novo exame físico com aferição da temperatura, ausculta pulmonar e cardíaca, verificação de linfonodos e mucosas, estando todos os parâmetros dentro da normalidade. Foram coletadas novas amostras sanguíneas para nova análise.

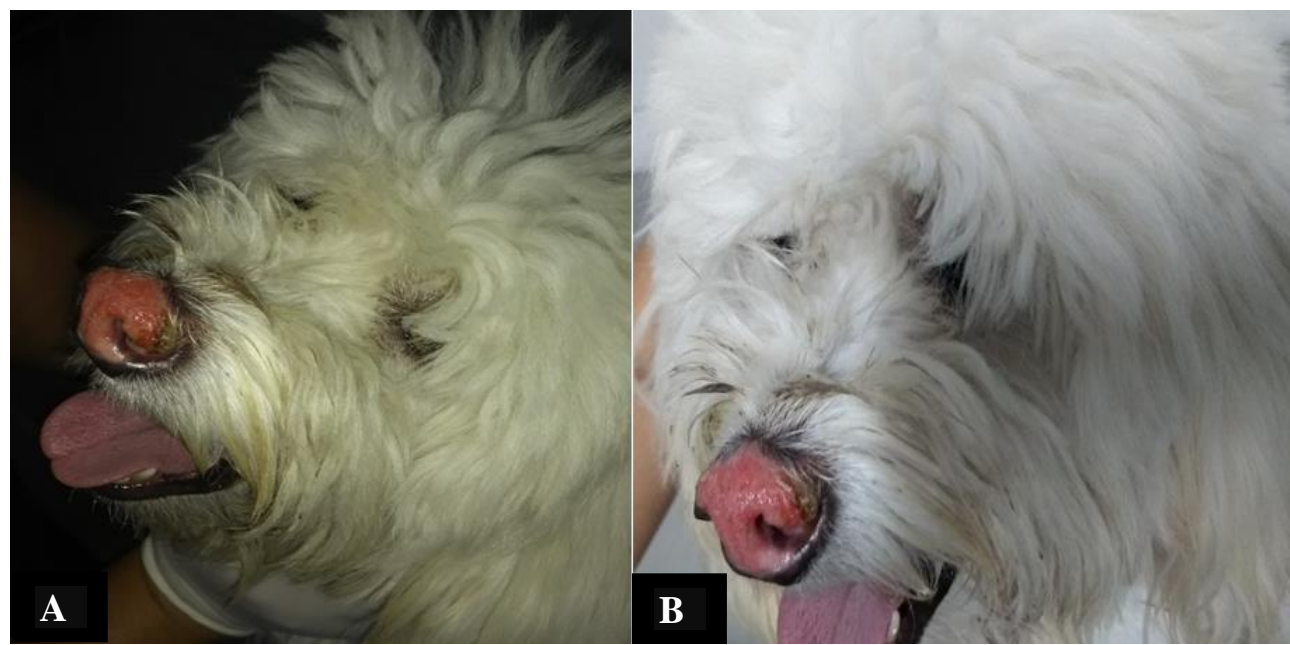

Figura 2. Retornos em A e B, no dia 20/09/2017 onde observa-se melhor arquitetura do plano nasal, diminuição da área crostosa, mas persistência da vermelhidão.

A escolha do tratamento foi realizada de acordo com a literatura que descreve a terapia imunossupressora deve-se basear-se no uso da prednisona ou prednisolona, na dose de 2,5 $\mathrm{mg} / \mathrm{kg}$, por via oral, SID, por 30 dias (Miller et al., 2013). Diante de melhora no quadro imunológico do animal (Tabela 1), a dose de corticosteroides foi aumentada para 2,5 $\mathrm{mg} / \mathrm{kg} / \mathrm{dia}$, por um período de 30 dias, onde será feita nova avaliação e $o$ restante dos medicamentos foram mantidos. Além da prescrição do uso de silimarina na dose de 22,5 $\mathrm{mg} / \mathrm{kg} / \mathrm{dia}$ para controle dessa alteração no exame bioquímico da enzima hepática. O restante do tratamento foi mantido para melhor resposta do animal.

$\mathrm{O}$ animal não retornou para novas análises e avaliação clínica, acredita-se que ainda se encontra em tratamento ou tenha melhorado as lesões, já que, o proprietário não retornou com o cão. O animal vinha apresentando bom estado físico e imunológico, com considerável melhora clínica da lesão, embora o processo de cicatrização ocorra lentamente devido ao local onde se encontra a mesma, que fica sempre exposta a radiação UV e a saliva do animal que constantemente se lambe em razão do incômodo. Sabe-se que a dermatopatia imunossupressora tem tratamento longo e duradouro. Normalmente, 
os cães que se encontram em terapia de manutenção, em um período de tempo de oito a 12 meses, devem ter o tratamento interrompido para julgamento, onde a não recorrência dos sinais clínicos pode ocorrer (Miller et al., 2013).

\section{Conclusão}

Apesar das doenças autoimunes ainda serem pouco evidenciadas na clínica médica, elas vêm ganhando espaço. Assim, podendo ser por uma melhor busca do clínico em novas formas de diagnóstico ou por grande quantidade de informações a que o proprietário vem tendo acesso. Isso proporciona estar sempre procurando um diagnóstico definitivo e assim, melhor elucidação do caso. Podemos avaliar a importância do exame histopatológico para o diagnóstico definitivo nos casos do complexo lúpus eritematoso. Este é de grande valia na elucidação do caso clínico, podendo ter-se feito o descarte de outras dermatopatias e definido o diagnóstico, sendo assim possível iniciar um eficaz tratamento com um bom prognóstico, devido a um melhor direcionamento do paciente e dos medicamentos a serem utilizados. Assim, levando até o proprietário uma resposta definitiva, que traz satisfação e segurança ao mesmo para começar um longo tratamento, que pode haver complicações durante seu percurso.

Por conta de o proprietário não ter retornado para as avaliações durante o final do tratamento, não temos como ter um retorno positivo para o fim da terapêutica, supõe-se que o animal tenha obtido uma melhora significativa. Já que o prognóstico do LED em pacientes caninos é reputado como bom, porém, seu controle medicamentoso, será requerido, provavelmente, durante toda vida do animal.

\section{Referências Bibliográficas}

Cardoso, M. J. L., Machado, L. H. A., Melussi, M., Zamarian, T. P., Carnielli, C. M. \& Júnior Ferreira, J. C. M. 2011. Dermatopatias em cães: revisão de 257 casos. Archives of Veterinary Science, 16, 66-74.

Ettinger, S. \& Feldman, E. 2004. Tratado de medicina interna veterinária: doenças do cão e do gato. Guanabara Koogan, Rio de Janeiro.

Gerhauser, I., Strothmann-Lverssen, A. \& Baumgorner, W. 2006. A case of interface perianal dermatitis in a dog: is this aunsual manifestation of lupus erythematosus? . Veterinary Pathology, 43, 761-764.
Gomes, P. M. S. 2015. Lúpus eritematoso cutâneo: manifestações clínicas e análise laboratorial. Revista SUSTINERE, 3, 3-21.

Goo, M. J., Park, J. K., Hong, I. H., Yang, H. J., Yuan, D. W., Ki, M. R., Han, J. Y., Ji, A. R., Kim, T. H., Williams, B. H. \& Jeong, K. S. 2008. Discoid lúpus erythematosus (DLE) in a Sptiz Dog. he Journal of Veterinary Medical Science, 70, 633-635.

Griffies, J. D., Mendelsohn, C. L., Rosenkrantz, W. S. M., R., Boord, M. J. \& Griffin, C. E. 2004. Tropical 0, $1 \%$ tracolimus for the treatment of discoid Lupus erythematosus and Pemphigus erythematosus in dogs. Journal of the American Animal Hospital Association, 40, 29-41.

Gross, T. L., Ihrke, P. J., Walder, J. E. \& Affolter, K. V. 2009. Doenças de pele do cão $e$ do gato: diagnóstico clínico $e$ histopatológico. Roca, São Paulo.

Larsson, C. E. 1996. Dermatopatias alérgicashipersensibilidade medicamentosa (HM). Clínica Veterinária, 5, 351-355.

Larsson, C. E. \& Otsuka, M. 2000. Lúpus eritematoso discóide - LED: Revisão e casuística em serviço especializado na capital de São Paulo. Revista de Educação Continuada do CRMV-SP, 3, 29-36.

Miller, W. H., Griffin, C. E., Campbell, K. L. \& Muller, G. H. 2013. Small Animal Dermatology. Elsevier Health Sciences, St Louis.

Mueller, R. S. 2005. Immune-mediated skin diseases. $56^{\circ}$ Congresso Nazionale Multisala Scivac. Itália.

Patel, A. \& Forsythe, P. J. 2011. Dermatologia em pequenos animais. Elsevier Brasil, Rio de Janeiro.

Scott, D. W., Muller, G. H. \& Kirk, R. W. 1996. Dermatologia dos pequenos animais. Interlivros, Rio de Janeiro.

Tizard, I. R. 2002. Imunologia Veterinária: introdução. Roca, São Paulo, Brasil.

Val, A. C. 2006. Doenças cutâneas autoimunes e imunomediadas de maior ocorrência em cães e gatos: revisão de literatura. Clínica Veterinária, 60, 68-74.

Article History:

Received 7 November 2017

Accepted 20 December 2017

Available online 28 February 2018

License information: This is an open-access article distributed under the terms of the Creative Commons Attribution License 4.0, which permits unrestricted use, distribution,and reproduction in any medium, provided the original work is properly cited. 